\title{
Multiplicative modelling of four-phase microbial growth
}

\author{
María Jesús Munoz-Lopez ${ }^{1,2 *}$, Maureen P. Edwards ${ }^{3}$, Ulrike Schumann ${ }^{4,5}$ and Robert S. Anderssen 6
}

\begin{abstract}
Microbial growth curves, recording the four-phases (lag, growth, stationary, decay) of the dynamics of the surviving microbes, are regularly used to support decision-making in a wide variety of health related activities including food safety and pharmaceutical manufacture. Often, the decision-making reduces to a simple comparison of some particular feature of the four-phases, such as the time at which the number of surviving microbes reaches a maximum. Consequently, in order to obtain accurate estimates of such features, the first step is the determination, from experimental measurements, of a quantitative characterization (model) of the four-phases of the growth-decay dynamics involved, which is then used to determine the values of the features. The multiplicative model proposed by Peleg and colleagues is ideal for such purposes as it only involves four parameters which can be interpreted biologically. For the determination of the four parameters in this multiplicative model from observational data, an iterative two-stage linear least squares algorithm is proposed in this paper. Its robustness, which is essential to support successful comparative assessment, is assessed using synthetic data and validated using experimental data. In addition, for the multiplicative model, an analytic formula is derived for estimating the average lifetimes of the surviving microbes.
\end{abstract}

\section{Introduction}

For microbial growth considerations in areas as diverse as food contamination and pharmaceutical manufacture, the key data are the four-phases (lag, growth, stationary, decay) of the growth dynamics of the surviving microbes $[7,8]$. For the utilization of such data for comparative assessment, monitoring and predictive purposes, an appropriate model is required which accurately tracks the four phases [8]. Depending on the situation under consideration, such a model can be utilized in various ways. In food contamination situations, it can be used to compare different inactivation (heating) strategies in food processing or to comparatively assess the survival characteristics of different classes of microbes. In pharmaceutical situations, it can be used to predict the optimal time to harvest the surviving microbes, since it is only the surviving microbes that can be used to make the pharmaceutical. In the study of soil microbes, comparative assessment has been used to compare the chemical and physical factors

\footnotetext{
*Correspondence: munozlom@tcd.ie

'Mathematical Sciences Institute, Australian National University, Canberra, ACT 2601, Australia

2Present address: School of Mathematics, Trinity College Dublin, Dublin 2,

Ireland

Full list of author information is available at the end of the article
}

which influence the relative levels of microbial carbon and nitrogen biomasses [10].

For modelling and tracking the changing features of the four-phases, Peleg and colleagues $[8,9]$ have proposed and analysed a multiplicative model consisting of the product of two Kohlrausch (stretched exponential) functions [2, 7] with positive and negative exponential growth

$$
N(t)=N_{0} \exp \left[\left(\frac{t}{t_{c g}}\right)^{m_{1}}\right] \exp \left[-\left(\frac{t}{t_{c d}}\right)^{m_{2}}\right]
$$

where the parameters $t_{c g}$ and $t_{c d}$ represent the characteristic times for the growth and the decay, respectively, (had they been unimpeded) and the exponents $m_{1}$ and $m_{2}$ characterize the nature of the exponential growth and decay. Such a model, after the initial growth from a starting population of $N_{0}$, allows for a subsequent decrease in the size of the population, as occurs for the survivors in a closed environment $[7,8]$. On setting $m_{1}=\beta, m_{2}=b$, $\alpha=\left(1 / t_{c g}\right)^{m_{1}}$ and $a=\left(1 / t_{c d}\right)^{m_{2}}$, Eq. (1) takes the more compact form

$$
N(t)=N_{0} \exp \left(\alpha t^{\beta}\right) \exp \left(-a t^{b}\right)
$$

\section{Springer}


which models an initial growth (by having $\alpha>a$ ) which is eventually dominated by the decay (by having $b>\beta$ ).

It is easier to describe the algorithm using this equation. Once the parameters $a, b, \alpha$ and $\beta$ have been determined, one can then use the above relationships to determine $m_{1}, m_{2}, t_{c g}$ and $t_{c d}$. These relationships are discussed from a biological interpretative perspective in subsections 2.3 and 2.4.

As explained in Edwards et al. [7], the importance of this model is that it is the solution of a non-autonomous ordinary differential equation which is able to track the four-phases. It therefore circumvents the shortcomings associated with models which are the solutions of autonomous ordinary differential equation, such as the Verhulst, since their solutions can only model the first, second and third phases, but not the fourth.

To determine the parameters in fitting the multiplicative model to observational survival data, Peleg and Corradini [8] suggest the use of mathematical software such as Mathematica. The challenge here is the need to find starting values for the parameters which are representative of the situation under consideration and to ensure that the solver used is stable with respect to measurement noise and limited data.

Here, it is shown how the special structure of the multiplicative model can be exploited to derive an iterative two-step procedure for the determination of the parameters. An assessment of its robustness, using synthetic data, is given. Validation is performed using microbial (fungal) survival measurements.

The paper has been organized in the following manner. The multiplicative model is discussed in Section 2 and an analytic average lifetime formula for it is derived. The algorithm is proposed in Section 3 and tested on synthetic data in Section 4. The application of the algorithm to real microbial survival data is the subject of Section 5 along with conclusions.

\section{The model}

The multiplicative model proposed by Peleg et al. (2009) [9] can be derived in various ways.

\subsection{From first principles}

For an initial population $N_{0}>0$, unrestrained growth can be modelled as a positive exponent stretched exponential (Kohlrausch) function

$$
N_{g}(t)=N_{0} \exp \left[\left(\frac{t}{t_{c g}}\right)^{m_{1}}\right]
$$

where $t_{c g}$ denotes the characteristic growth time and $m_{1}$ characterizes the rate of growth.
The decay can be modelled in a similar manner as a negative exponent stretched exponential (Kohlrausch) function

$$
f_{d}(t)=\exp \left[-\left(\frac{t}{t_{c d}}\right)^{m_{2}}\right], \quad 0 \leq f_{d}(t) \leq 1, m_{2}>m_{1}
$$

where $t_{c d}$ denotes the characteristic decay time and $m_{2}$ characterizes the rate of decay.

If it is assumed that the decay modifies the growth multiplicatively as a function of the time, then Eqs. (3) and (4) combine to give

$$
N(t)=N_{g}(t) f_{d}(t)
$$

or, equivalently,

$$
N(t)=N_{0} \exp \left[\left(\frac{t}{t_{c g}}\right)^{m_{1}}\right] \exp \left[-\left(\frac{t}{t_{c d}}\right)^{m_{2}}\right] .
$$

Justification for this being a realistic model of a fourphase growth-decay process is given in Edwards et al. [7], where it is shown that such a structure corresponds to the solution of a non-autonomous ordinary differential equation model of a quite general growth-decay process.

\subsection{Solution of the non-autonomous von Bertalanffy equation}

As noted by Edwards et al. [7], a key property of the multiplicative model (2) is that it is a solution of the non-autonomous von Bertalanffy equation

$$
\frac{d N}{d t}=\bar{\alpha}(t) N^{\bar{\beta}}-\bar{a}(t) N^{\bar{b}}+\psi(t), N(0)=N_{0}, \bar{\beta}>0, \bar{b}>0,
$$

when $\bar{\alpha}(t)=\alpha \beta t^{\beta-1}, \bar{\beta}=1, \bar{a}(t)=a b t^{b-1}, \bar{b}=1$ and $\psi(t)=0$. On substituting these values into (5) and setting $\psi(t)=0$, the last equation becomes

$$
\frac{d N}{d t}=\theta(t) N, \quad \theta(t)=\left[\alpha \beta t^{\beta-1}-a b t^{b-1}\right],
$$

which becomes, when $\theta(t)$ is a constant because $\beta=b=$ 1 , the standard exponential growth-decay equation.

For the von Bertalanffy Eq. (5), Edwards and Anderssen [6] have performed a Lie point symmetry analysis to identify the regularity that $\bar{\alpha}, \bar{\beta}, \bar{a}, \bar{b}$ and $\psi(t)$ must satisfy in order for (5) to have interesting classes of analytic solutions (often referred to technically as non-trivial symmetries). Such symmetries can then be utilized to explore for new closed form solutions.

\subsection{Biological interpretation of the parameters $\alpha, \beta, a$ and $b$}

The relevance of the above two derivations for Eqs. (1) and (2) is that they shed light on how to interpret the parameters $\alpha, \beta, a$ and $b$ biologically and study their interactive 
interdependence. The starting point is Eq. (2) rewritten in its equivalent form (6).

For the standard decay Eq. (6), when $\theta(t)$ is a constant $\theta_{0}$ and the population corresponds to a discrete ensemble of members, as holds for microbial growth-decay, the characteristic time of the exponential decay $1 / \theta_{0}$ corresponds algebraically to the "mean lifetime", $\ell_{0}$, of the members in the ensemble. (The algebraic details are contained in the Appendix.) In addition, if all the individual lifetimes are measured with respect to the same initial reference state, then $1 / \theta_{0}$ corresponds to the arithmetic mean of these individual times.

As highlighted in the Appendix, the mean lifetime concept can be extended to any four-phase microbial growthdecay situation which decays to zero. This generalized mean lifetime will be denoted by $\ell_{\theta}$. Its importance relates to the fact that it measures a key biologically relevant feature, the average life time of the microbes in a four-phase growth-decay situation. From a food safety perspective, $\ell_{\theta}$ can be used to identify strategies that allow inactivation to be performed effectively, whereas, from a pharmaceutical perspective, an understanding of the value of $\ell_{\theta}$ is required to guarantee the time optimal harvesting of live microbes.

The corresponding formula for the $\theta(t)$ of Eq. (6) thereby takes the form

$$
\begin{aligned}
\ell_{\theta}=\ell_{\alpha, \beta, a, b}= & \int_{0}^{\infty} \exp \left(\alpha \tau^{\beta}-a \tau^{b}\right) \tau d \tau / \\
& \int_{0}^{\infty} \exp \left(\alpha \tau^{\beta}-a \tau^{b}\right) d \tau .
\end{aligned}
$$

In particular, with respect to given growth-decay data, the algorithm is used to determine the values of the parameters $\alpha, \beta, a$ and $b$ which are then substituted into Eq. (7) which can be evaluated using Matlab.

As is clear from the form of the right hand side of Eq. (7), the value of $\ell_{\alpha, \beta, a, b}$ can be used to compare different scenarios for the parameters $\alpha, \beta, a$ and $b$. For example, since together $\alpha$ and $\beta$ identify how a particular microbial population grows, the values of $\alpha$ and $\beta$ could be fixed and the values of $a$ and $b$ varied to find the minimum value of the average lifetimes $\ell_{\alpha, \beta, a, b}$ as a characterization for an optimal strategy for performing inactivation.

Comparative values for $\ell_{\alpha, \beta, a, b}$ for various growth-decay dynamics are discussed in subsection 5 .

\subsection{Biological interpretation of the parameters $t_{c g}, t_{c d}, m_{1}$ and $m_{2}$}

If $m_{1}=m_{2}=1$, the parameters $t_{c g}$ and $t_{c d}$ correspond, respectively, to the characteristic times of the growth and decay. In particular, they characterize how quickly the growth and decay of the microbes within a population occur, with the rate of growth (decay) being inversely proportional to the value of $t_{c g}\left(t_{c d}\right)$. Consequently, the values of $t_{c g}$ and $t_{c d}$ give an immediate indicative illustration of the relative strengths of the growth and decay dynamics.

However, the interpretation of the contributions of $t_{c g}$ and $t_{c d}$ to the growth-decay dynamics must be modified by the values of $m_{1}$ and $m_{2}$. Since $m_{1}=\beta$ and $m_{2}=b$, it follows, on equating coefficients in Eqs. (1) and (2), that

$$
t_{c g}=\alpha^{-1 / \beta}=\frac{1}{\alpha^{1 / \beta}} \quad \text { and } \quad t_{c d}=a^{-1 / b}=\frac{1}{a^{1 / b}} .
$$

Consequently, multiple choice of $\alpha$ and $\beta$ ( $a$ and $b$ ) will generate the same value for $t_{c g}\left(t_{c d}\right)$. Such ambiguities are resolved by determining $\alpha$ and $\beta$ ( $a$ and $b$ ) from the experimental data of the growth-decay dynamics under consideration. The linear least squares procedures, as outlined in section 3.1, achieve this by first estimating the values of $\alpha$ and $\beta$, and then the values of $a$ and $b$, separately in an iterative manner.

\subsection{Properties of the multiplicative model}

Sufficient conditions, in terms of the parameters in the more compact form (2) for the multiplicative model, which guarantee a four-phase structure, are given by $\alpha>$ $a$ (which guarantees initial growth) and $b>\beta$ (which guarantees subsequent decay). Alternatively, in terms of the general form of Eq. (6) with an arbitrary $\theta$, four-phase dynamics is guaranteed if $\theta(t)$ is initially positive, which guarantees initial growth, and $\int_{0}^{\infty} \theta(\tau) d \tau=-\infty$, which guarantees that subsequent decay occurs and goes to zero.

Taking logarithms of the more compact form (2) yields the additive relationship

$$
\ln N(t)=\ln N_{0}+\alpha t^{\beta}-a t^{b},
$$

or

$$
\ln N(t)-\ln N_{0}=\alpha t^{\beta}-a t^{b},
$$

which will play a key role in the formulation of the algorithm.

Consequently, the logarithmic growth-decay dynamics, at a given time $t_{*}$, corresponding to the number of surviving microbes at that time, thereby becomes

$$
\left.\frac{d \ln N(t)}{d t}\right]_{t^{*}}=\alpha \beta t_{*}^{\beta-1}-a b t_{*}^{b-1},
$$

which yields a connection back to the multiplicative model being the solution of a particular form of the von Bertalanffy equation.

\section{The algorithm}

The essence of the current situation is the fitting of the multiplicative model (2) to given experimental data, which reduces to the determination of estimates for the four parameters $\alpha, \beta, a, b$. However, the multiplicative model is non-linear and the amount of experimental data 
available is usually quite limited. The standard procedure proposed by various authors is to use some non-linear regression software package such as is available in Matlab. The limitation here is the need to find starting values for the parameters which are representative of the situation under consideration. In comparative assessment situations, it is important that the estimates of the values of the parameters $\alpha, \beta, a, b$ correctly characterize the situations being compared. For example, if the value of the parameter $b$ was used to assess the effectiveness of different inactivation strategies, then the estimates of $b$, utilized for the comparative assessment, must correctly represent the actual decay occurring so that no incorrect action or advice was implemented.

As explained below, because of the way in which the estimation is performed, the determination of the parameters $\alpha, \beta, a, b$ is essentially unique in that the estimation is performed, iteratively, as two separate steps involving first the growth phase, to determine $\alpha$ and $\beta$, and then the decay phase, to determine $a$ and $b$.

In a sense, compared with non-linear least squares methods, the proposed algorithm is an example of "let the data decide". The rationale is that if one just uses a non-linear solver to do the parameter identification, then no specific structure is exploited within the data which relates to subsets of the parameters. In the algorithm proposed here, this is possible as, in the multiplicative model, the model is separable into a growth component, involving only $\alpha$ and $\beta$, and a decay component, involving only $a$ and $b$.

\subsection{Estimating the parameters}

In the past, different methods have been proposed and used to model microbial growth and decay dynamics.

For instance, in order to assess the nature of the initial lag-phase of growth-decay dynamics, Baranyi et al. [3] proposed the use of detection times. However, this requires that the detection times be limited to the initial exponential growth in order to avoid underestimating the rate of growth of the lag-phase.

A different suggestion, proposed by Baranyi and colleagues $[4,5]$, was to solve a time separable nonautonomous model of the form

$$
\frac{d N(t)}{d t}=\phi(t) \mu(N) N, \quad N(0)=N_{0},
$$

where the separable time function $\phi(t)$ performs the transformation of the autonomous equation

$$
\frac{d N(t)}{d t}=\mu(N) N, \quad N(0)=N_{0},
$$

into the non-autonomous Eq. (11). Various choices for $\phi(t)$ have been proposed and analysed by Baranyi and colleagues. However, they have not chosen a form for $\phi(t)$ that corresponds to that for the non-autonomous equation which generates the multiplicative model (2). In particular, their emphasis is on modelling the growth of the total population.

Peleg and Corradini [8], for determination of the parameters in the multiplicative model (1), suggest non-linear least squares. The difficulty is that representative starting values for the parameters must be chosen for the implementation of such methods, which the proposed algorithm avoids.

The algorithm proposed and implemented here, which explicitly exploits the properties of logarithms, is based on the iterative use of two linear least squares approximations applied to different phases of a growth curve. Its advantage is that it can be iterated to obtain successively better approximations for the parameters $\alpha, \beta, a, b$. This type of algorithm does not appear to have been published in the microbial growth modelling literature, though it has been used to determine the parameters of the stretched exponential (Kohlrausch) function in rheological and biological applications [1].

Consider the model in the form

$$
N(t)=N_{0} \exp \left(\alpha t^{\beta}\right) \exp \left(-a t^{b}\right) \text {. }
$$

On taking the logarithm and reorganizing, the last equation becomes

$$
\ln N(t)-\ln N_{0}=\alpha t^{\beta}-a t^{b} .
$$

For the initial growth data, the decay term $\exp \left(-a t^{b}\right)$ can be neglected, since it is the behaviour of $\exp \left(\alpha t^{\beta}\right)$ that dominates at this stage. Consequently, the first step in the implementation of the algorithm is the determination of initial estimates $\alpha_{1}$ and $\beta_{1}$ for $\alpha$ and $\beta$ using the model

$$
\ln \left\{\ln N(t)-\ln N_{0}\right\}=\ln \alpha+\beta \ln t .
$$

With respect to a representative sample $d_{i}^{*}=N\left(t_{i}\right), i=$ $1,2, \cdots, I, I>>2$, of the first two of the four-phases, a linear least squares estimate can be derived for $\ln \alpha_{1}$, and hence $\alpha_{1}$, and $\beta_{1}$ using the overdetermined system of equations

$$
\ln \left[\ln d_{i}^{*}-\ln N_{0}\right]=\ln \alpha+\beta \ln t_{i}, \quad i=1,2, \ldots, I .
$$

The second step in the implementation of the algorithm is the determination of initial estimates $a_{1}$ and $b_{1}$ for $a$ and $b$ using the model

$$
\ln \left\{-\ln N(t)+\ln N_{0}+\alpha_{1} t^{\beta_{1}}\right\}=\ln a+b \ln t .
$$

With respect to a representative sample $d_{j}^{\#}=N\left(t_{j}\right), j=$ $1,2, \cdots, J, J>>2$, of the last two of the four-phases, a linear least squares estimate can be derived for $\ln a_{1}$, 
and hence $a_{1}$, and $b_{1}$ using the overdetermined system of equations

$$
\begin{aligned}
& \ln \left[-\ln d_{j}^{\#}+\ln N_{0}+\alpha_{1} t_{j}^{\beta_{1}}\right] \\
& \quad=\left[\ln a_{1}+b_{1} \ln t_{j}\right], j=1,2, \ldots, J .
\end{aligned}
$$

The third step in the implementation of the algorithm is the determination of estimates $\alpha_{2}$ and $\beta_{2}$ for $\alpha$ and $\beta$ using the model

$$
\ln \left\{\ln N(t)-\ln N_{0}+a_{1} t^{b_{1}}\right\}=\ln \alpha+\beta \ln t .
$$

With respect to a representative sample $d_{\ell}^{*}=N\left(t_{\ell}\right), \ell=$ $1,2, \cdots, L, L>>2$, of the lag and growth phases, a linear least squares estimate can be derived for $\ln \alpha_{2}$, and hence $\alpha_{2}$, and $\beta_{2}$ using the overdetermined system of equations

$$
\ln \left[\ln d_{\ell}^{*}-\ln N_{0}+a_{1} t_{\ell}^{b_{1}}\right]=\ln \alpha_{2}+\beta_{2} \ln t_{\ell} .
$$

The fourth, fifth, $\cdots$ steps in the implementation now iterate, respectively, between the second and third steps.

\subsection{Algorithm implementation}

Because the implementation of the algorithm involves the evaluation of logarithms, the choice of the scale for the times becomes an important issue. In the situations studied here, the basic time scale is days.

However, for measurements made at fractions of a day, the logarithms will be negative. Consequently, to avoid this potential difficulty, it is best to work with a time scale (hours, minutes or seconds) such that all the times, at which measurements were made, are greater than one.

\section{The validation of the algorithm using synthetic data}

The numerical performance of the algorithm was initially assessed using the following uniform grid discrete synthetic data

$$
\begin{aligned}
\left\{N\left(t_{i}\right)\right\}= & \left\{N\left(t_{i}\right)=N_{0} \exp \left(\alpha t_{i}^{\beta}\right)\right. \\
& \left.\times \exp \left(-a t_{i}^{b}\right) \mid i=1,2, \cdots, I\right\},
\end{aligned}
$$

where the values of $N_{0}$ and the parameters $\alpha, \beta, a, b$ are specified. The discrete values $\left\{N\left(t_{i}\right)\right\}$ were used to simulate exact and non-exact measurements scenarios of the four-phase growth-decay dynamics with the goal of testing the performance of the algorithm with respect to

$\{1\}$ the accuracy of the recovery of the parameters, and

\{2\} the quality of the reconstructions of the four-phase growth-decay dynamics curves compared with the actual $N(t)$.

Though the comparison of the reconstructions of the growth-decay dynamics is indicatively important, the key issue is the robustness, accuracy and reliability of the recovery of the parameters, as it is those that will be used for subsequent decision-making and comparative assessments.

\subsection{The synthetic data analysis}

The exact synthetic data used to test the algorithm was generated using the discrete multiplicative model data $\left\{N\left(t_{i}\right)\right\}$ of Eq. (20) with the parameter values $\alpha=6, \beta=$ $1.5, a=4, b=2$ and $N_{0}=100$.

\subsubsection{Exact synthetic data inversion}

For the synthetic data situation without noise, only 7 data points are needed to perform the parameter estimation using the algorithm, which returns the correct values $\alpha=$ $6, \beta=1.5, a=4, b=2$.

The result for the exact data situation is illustrated in Fig. 1, where the exact and estimated curves are recovered exactly.

\subsubsection{Simulation studies of non-exact synthetic data inversion}

It is known that, in carefully performed measurements of microbial growth-decay dynamics, the measurement error does not depend on the size of the population as it evolves. Consequently, it was only necessary to test the robustness of the algorithm with respect to Gaussian error perturbations.

For this, the exact discrete values $\left\{N\left(t_{i}\right)\right\}$ were perturbed in the following manner to generate the simulated measurement data

$d_{i}^{(G)}=N\left(t_{i}\right)+K \epsilon_{i}, i=1,2, \cdots, 100, K \sim$ constant

with the $\left\{\epsilon_{i}\right\}$ being Gaussian errors with mean zero and variance 1 . In order to comprehensively test the performance of the algorithm, the inversion was performed on

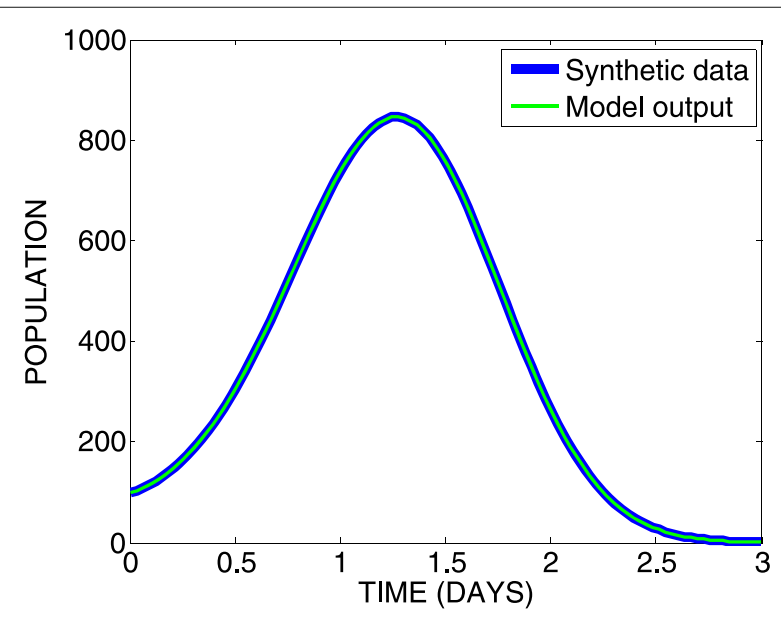

Fig. 1 Reconstruction for exact synthetic data 
500 realizations of the simulated measurement data $d_{i}^{(G)}$ with the corresponding values of $\alpha, \beta, a, b$, thereby generated, summarized as histograms as in Fig. 2.

As is clear from Fig. 2, the level of uncertainty in the determination of the parameters $\alpha, \beta, a, b$ increases as the level of the added Gaussian errors increases. As is clear from Fig. 1, the range of the values of $N(t)$ is approximately $\sim[0,800]$. Consequently, it is only when the value of $K$, relative to $N(t)$, becomes suitably large that a spread in the values of $\alpha, \beta, a, b$ becomes graphically significant. In addition, it shows that the values of the exponents $\beta$ and $b$ are more accurately recovered than the multipliers $\alpha$ and $a$. This difference in the recovery of $\beta$ and $b$, compared with that for $\alpha$ and $a$, is confirmed in terms of the statistics of the values of the parameters $\alpha, \beta, a, b$ tabulated in Fig. 3.

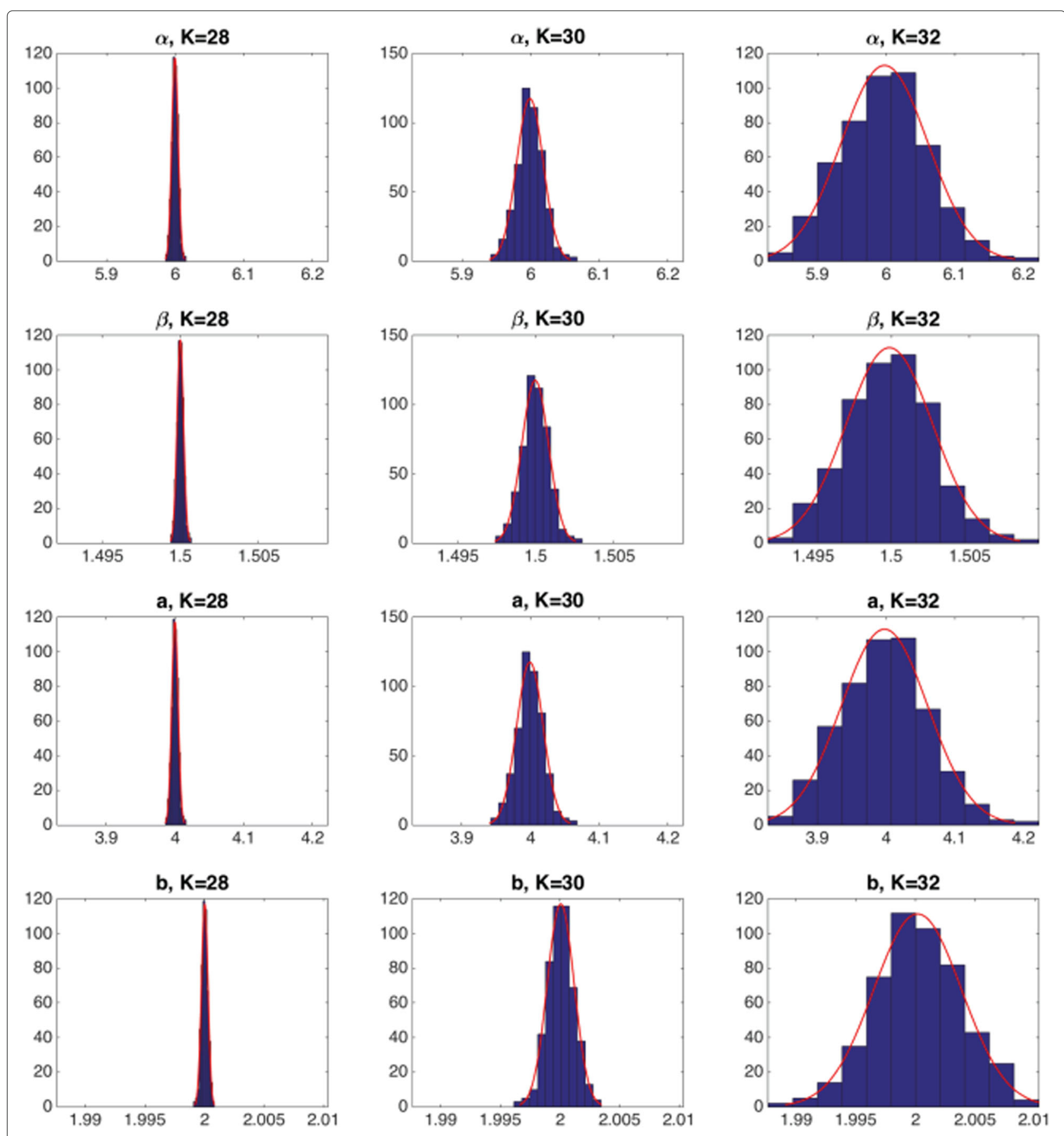

Fig. 2 Histograms of the parameter value $\alpha, \beta, a, b$ for different levels of the added Gaussian errors for 500 realizations 


\begin{tabular}{|l|r|c|c|}
\hline \multicolumn{1}{|c|}{ 100 data points } & $\mathrm{K}$ & $\begin{array}{c}\text { Mean Estimate } \\
\text { (over 500 runs) }\end{array}$ & Standard Deviation \\
\hline alpha & 28 & 5.99975 & 0.00463017 \\
\hline (original value: 6) & 30 & 5.99903 & 0.0193903 \\
\hline beta & 32 & 5.99775 & 0.0631314 \\
\hline (original value: 1.5) & 28 & 1.49999 & 0.000203996 \\
\hline a & 30 & 1.49995 & 0.00085432 \\
\hline (original value: 4) & 32 & 1.49987 & 0.00277303 \\
\hline b & 28 & 3.99975 & 0.00462315 \\
\hline (original value: 2 ) & 30 & 3.99903 & 0.0193609 \\
\hline
\end{tabular}

Fig. 3 The statistics of the parameters $\alpha, \beta, a, b$ for different levels of the added Gaussian errors for 500 realizations. The standard deviations for $\beta$ and $b$ are considerably less than those for $\alpha$ and $a$

This difference represents a direct illustration of how fundamental $\beta$ and $b$ are to determining the growth and decay, respectively, in order to accurately recover a fourphase structure. It implies that a good fit to a four-phase structure cannot be achieved by simply varying $\alpha$ and $a$ unless good estimates of $\beta$ and $b$ have been determined. This interpretation is implicit in the proposed algorithm, as illustrated in Eqs. (19) and (17), which highlight that $\beta$ and $b$ are the slopes of the straight lines that are fitted to the logarithmic data. This relates to the fact that, in

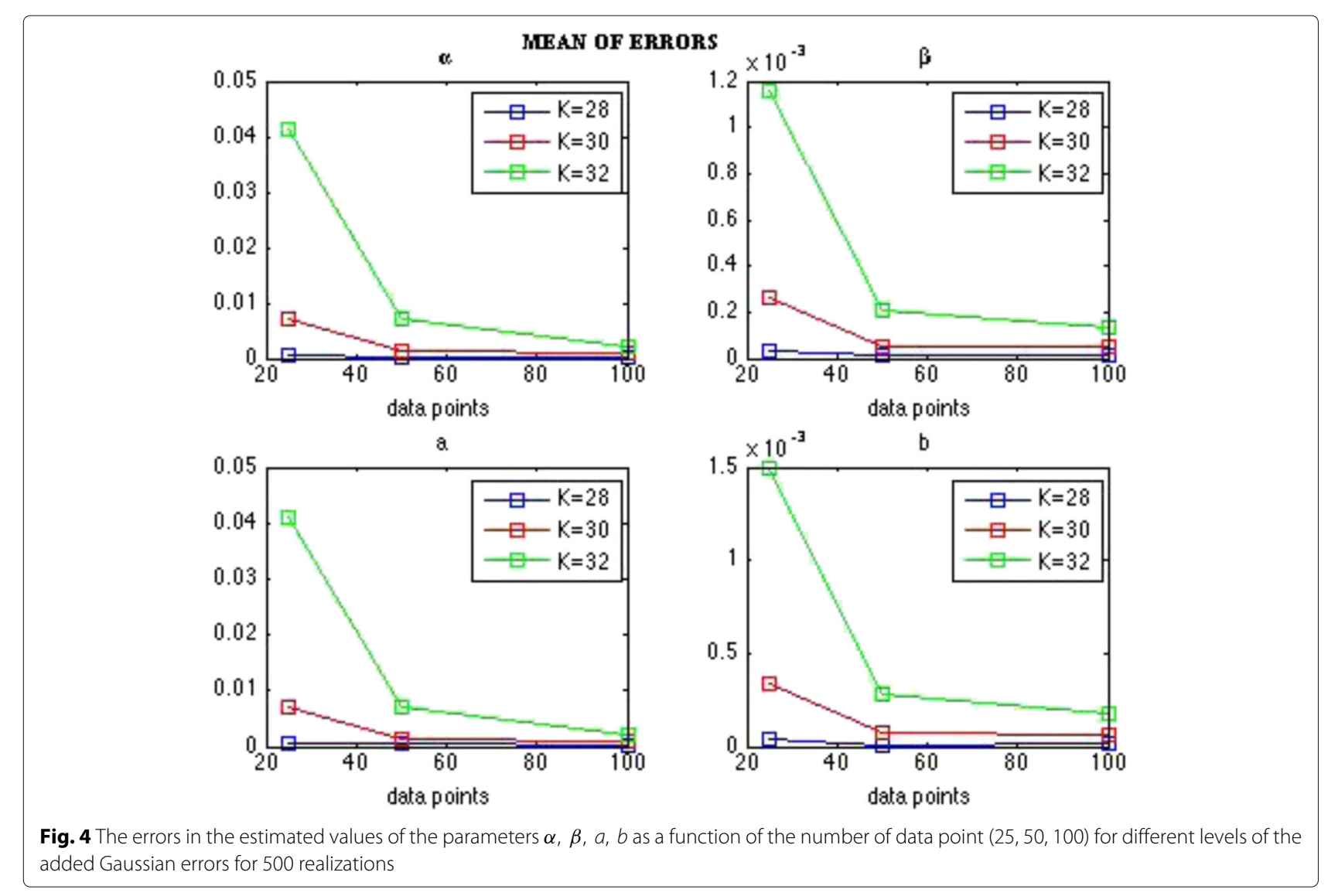



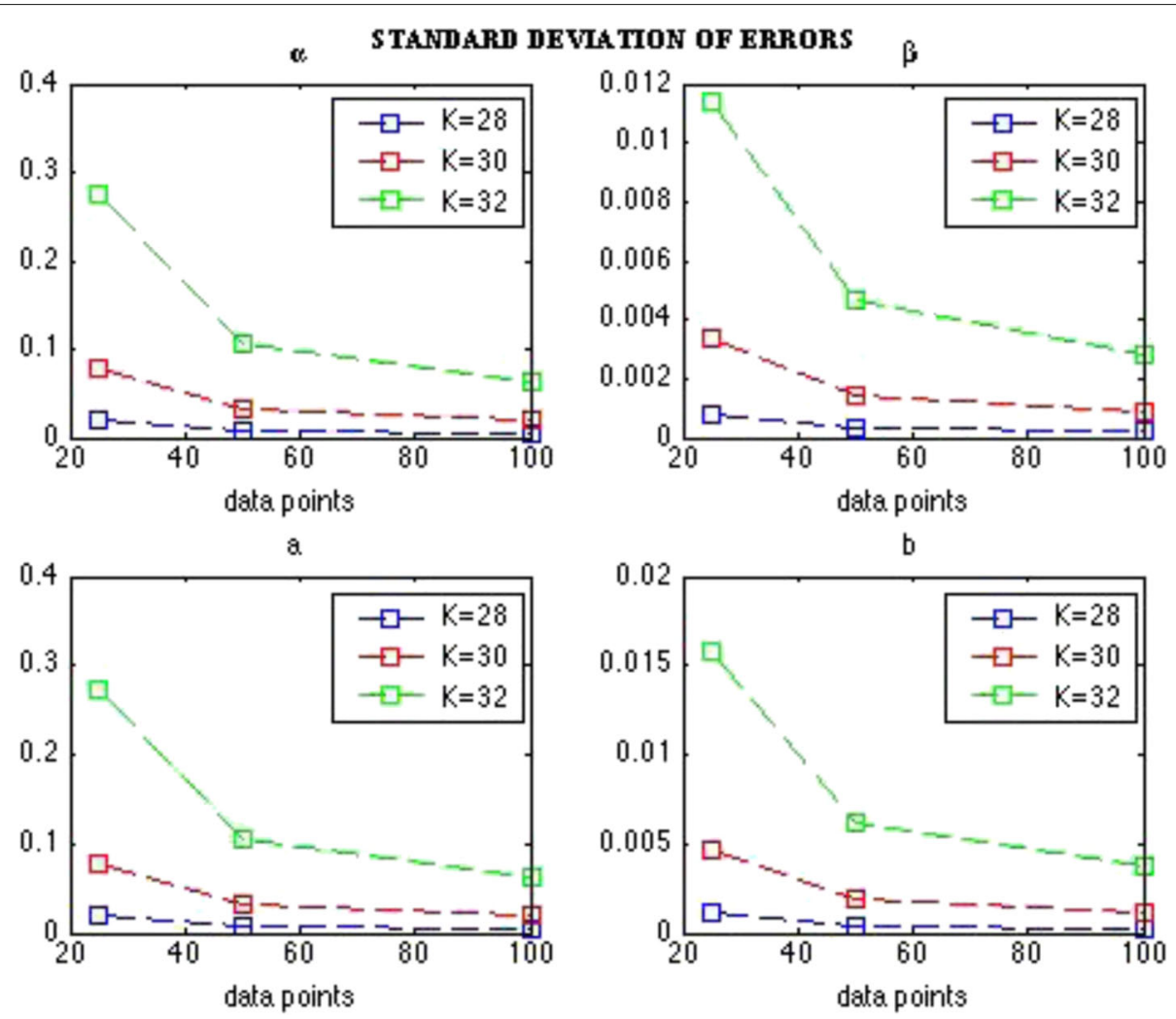

Fig. 5 The standard deviations of the errors in the estimated values of the parameters $\alpha, \beta, a, b$ as a function of the number of data point (25, 50, 100) for different levels of the added Gaussian errors for 500 realizations

terms of the linearity of the algebra of Eqs. (19) and (17), the constants $\ln (\alpha)$ and $\ln (a)$ do not influence the actual slopes $\beta$ and $b$ of the straight line fits to the logarithmic data.

Furthermore, the algorithm estimates $\beta$ and $b$ separately using, respectively, a growth component and a decay component of the four-phase structure. Consequently, this illustrates the uniqueness in the determination of the parameters $\alpha, \beta, a, b$ and, hence, the values of $t_{c g}$ and $t_{c d}$ of Eq. (8).

The importance of the number of data points used in the recovery of the parameters is illustrated in Figs. 4 and 5 . It shows that something like $\sim 50$ data points are required to guarantee reliable results. This highlights the difficulty of the often occurring practical situation of only having a small number of measurements (such as 10) of the growth-decay dynamics.

\section{Application of the algorithm to microbial survival data and conclusions}

5.1 Recovery of the parameters $\alpha, \beta, a, b$

In order to illustrate the practicality of the algorithm for real data, it was applied to the measurements from a study of the growth-decay dynamics for the filametus fungus Fusarium oxysporum.
Fusarium oxysporum is a plant pathogenic fungus with a wide host range causing a variety of diseases contributing to crop losses all over the globe. To obtain microbial growth data in a closed environment we monitored the growth of the fungus Fusarium oxysporum in minimal

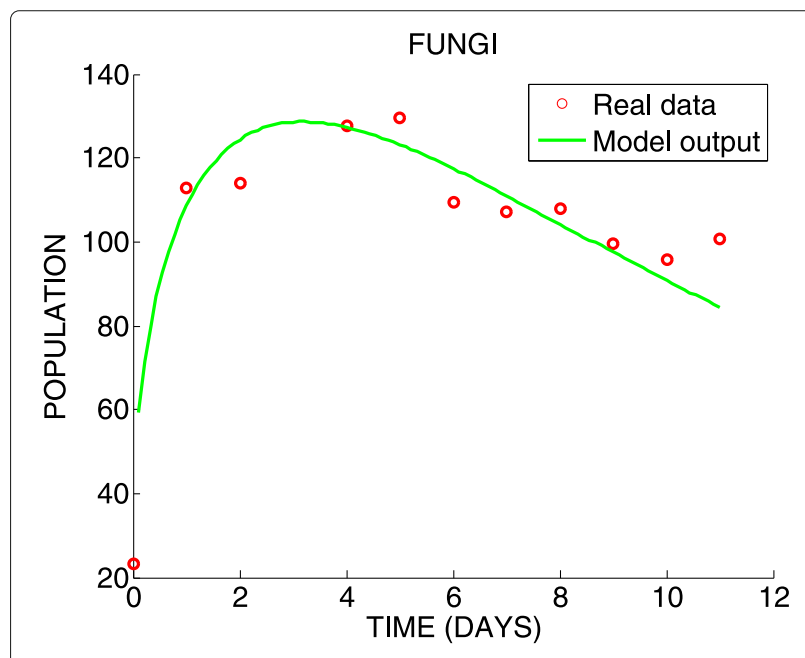

Fig. 6 The growth-decay dynamics for the fungus Fusarium oxysporum f.sp. conglutinans 

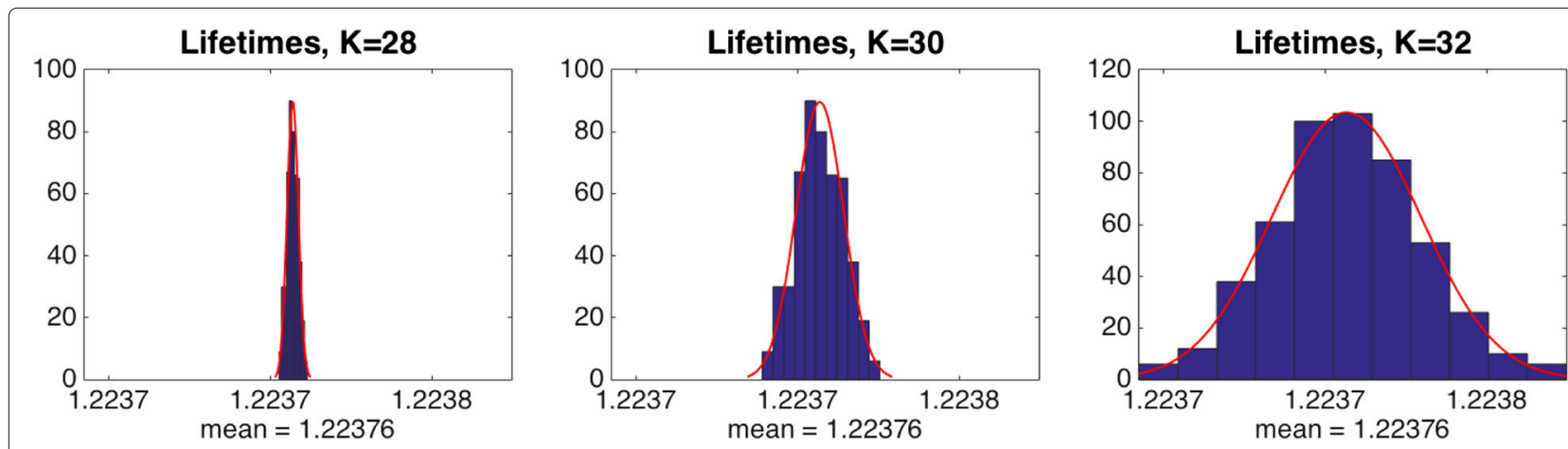

Fig. 7 Histograms for the values of $\ell_{\alpha, \beta, a, b}$ for different levels of the added Gaussian errors for 500 realizations using the synthetic data of Eq. (21)

media. A primary potato dextrose broth culture was inoculated with Conidiospores from a $-80{ }^{\circ} \mathrm{C}$ frozen stock and grown at $28{ }^{\circ} \mathrm{C}$, shaking at $200 \mathrm{rpm}$ for 2 days. Cells were collected by centrifugation, suspended in water, the optical density at $260 \mathrm{~nm}$ was measured and the cell concentration determined by comparison with a standard curve. A fresh secondary minimal medium culture was inoculated with $1.0 E 6$ cells $/ \mathrm{ml}$ and grown as above. In a temporal fashion, $1000 \mu \mathrm{l}$ samples were removed from the culture, the cells collected by centrifugation and suspended in water (between $100 \mu l$ and $500 \mu l$ ) adjusting the suspension volume as the culture became denser. Care was taken that cells were well suspended at all times by vigorous vortexing. Cells were then stained with Propidium Iodide for 5 minutes. Microscopic images were taken using three independent $5 \mu l$ subsamples imaging at least 7 independent regions of each sample. Bright field and fluorescence images were taken and the total number of cells counted using the bright field image. Dead cell counts were obtained from fluorescent images as propidium iodide permeates the membranes of dead cells staining these red. The average number of total and dead cells was determined and, as the cell suspension was more concentrated than the culture, the suspension volume was taken into account to determine the proportional number of total and dead cells in the culture.

The measurements represent a situation where the data is sparse and has only been measured for part of the decay phase. Nevertheless, it contains sufficient data to allow the algorithm to recover useful estimates of the parameters $\alpha, \beta, a, b$, which can be used to evaluate $\ell_{\theta}$ of Eq. (7).

\subsection{Evaluation of average lifetimes $\ell_{\alpha, \beta, a, b}$}

The generalized mean lifetime $\ell_{\alpha, \beta, a, b}$ of Eq. (7) was evaluated for the exact synthetic data of Fig. 1 and the fungus data of Fig. 6. The resulting values were 1.223757 and 14.14 days. Corresponding to the 500 simulations discussed above in relation to Figs. 1 and 2, the corresponding histogram for the resulting $\ell_{\alpha, \beta, a, b}$ values is plotted in Fig. 7. The means of the histograms in Fig. 7 are all the same for the three levels of noise considered, which represents indirect proof of the stability of $\ell_{\alpha, \beta, a, b}$. Its accuracy and reliability are reflected in the fact that these histogram means correspond to the rounding of the exact value of 1.223757.

\subsection{Conclusions}

For the determination of the four parameters $\alpha, \beta, a, b$ in the multiplicative model (2), a simple, easily implementable, iterative two-stage linear least squares algorithm has been proposed. Its robustness has been confirmed by testing it on synthetic data. Its practicality has been demostrated by applying it to measured growthdecay for the fungus Fusarium oxysporum.

In addition, for the multiplicative model, an analytic formula has been derived for estimating the average lifetimes $\ell_{\alpha, \beta, a, b}$ of the surviving microbes, which has been applied to the synthetic and measured data.

Overall, it appears that the numerical performance of the algorithm and the average liftime estimate will be useful in the support of decision-making related to health issues such as food safety and pharmaceutical manufacture.

\section{Appendix \\ Mean lifetime for microbial growth-decay for the multiplicative model \\ The standard decay model}

$$
\frac{d N}{d t}=-\lambda N, \quad N=N(t), \quad N(0)=N_{0}, \quad \lambda>0
$$

$\Downarrow$

$\Downarrow$ Solve:

$\Downarrow$

$$
N(t)=N_{0} \exp (-\lambda t), \quad N(\infty)=0
$$



distribution:

$\Downarrow$ Transform $N(t)$ to an exponential probability

$\Downarrow$

$$
\mathcal{P}(N(t))=\frac{\lambda}{N_{0}} N(t)=\lambda \exp (-\lambda t)
$$

$\Downarrow$

$\Downarrow$ The mean of the exponential distribution is $\lambda$ : $\Downarrow$

$$
\frac{1}{\lambda}=\tau=\text { relaxation time }=\text { mean lifetime }
$$

\section{The generalized decay model}

$$
\frac{d N_{\theta}}{d t}=\theta(t) N_{\theta}, \quad N_{\theta}(0)=N_{0}, \quad \theta(t)=\alpha \beta t^{\beta-1}-a b t^{b-1}
$$

$$
\begin{aligned}
& \Downarrow \\
& \Downarrow \text { Solve: } \\
& \Downarrow \\
& \quad N_{\theta}(t)=N_{0} \exp \left(\int_{0}^{t} \theta(\tau) d \tau\right), \quad N_{\theta}(\infty)=0
\end{aligned}
$$

$$
\Downarrow
$$$$
\Downarrow \text { Regularity: } \theta(0)>0 \text { and } \int_{0}^{\infty} \theta(\tau) d \tau=-\infty
$$$$
\Downarrow
$$$$
\Downarrow \text { Transform } N_{\theta}(t) \text { to a probability distribution: }
$$$$
\Downarrow
$$

$$
\mathcal{P}\left(N_{\theta}(t)\right)=\frac{N_{\theta}(t)}{\mathcal{A}\left(N_{\theta}(t)\right)}, \quad \mathcal{A}\left(N_{\theta}(t)\right)=\int_{0}^{\infty} N_{\theta}(\tau) \tau
$$

$\Downarrow$

$\Downarrow$ Compute the mean of $\mathcal{P}\left(N_{\theta}(t)\right)$ :

$\Downarrow$

$$
\mathcal{M}\left(\mathcal{P}\left(N_{\theta}(t)\right)\right)=\int_{0}^{\infty} \mathcal{P}\left(N_{\theta}(\tau)\right) \tau d \tau
$$

\section{Acknowledgements}

The authors thank the reviewer whose comments helped improved the clarity of the paper. The third author wishes to acknowledge the support of Prof. Thomas Preiss (Department of Genome Science, School of Medical Sciences (JCSMR), Australian National University, Garran Road, ACT 2601, Australia) for his support to carry out this research.

\footnotetext{
Author details

${ }^{1}$ Mathematical Sciences Institute, Australian National University, Canberra, ACT 2601, Australia. ${ }^{2}$ Present address: School of Mathematics, Trinity College Dublin, Dublin 2, Ireland. ${ }^{3}$ School of Mathematics and Applied Statistics, University of Wollongong, Wollongong 2522, NSW, Australia. ${ }^{4}$ CSIRO Plant Industry, GPO Box 1600, Canberra 2601, ACT, Australia. ${ }^{5}$ Present address: Department of Genome Science, School of Medical Sciences (JCSMR), Australian National University, Garran Road, Canberra 2601, ACT, Australia. ${ }^{6}$ CSIRO Digital Productivity, GPO Box 664, Canberra 2601, ACT, Australia.
}

Received: 26 May 2015 Revised: 28 August 2015

Accepted: 14 September 2015

Published online: 01 October 2015

\section{References}

1. Anderssen, R.S., Helliwell, C.A.: Information recovery in molecular biology: causal modelling of regulated promoter switching experiments. J. Math. Biol. 67, 105-122 (2013)

2. Anderssen, R.S., Husain, S., Loy, R.J.: The Kohlrausch function: properties and applications. ANZIAM J (E). 45, C800-C816 (2004)

3. Baranyi, J., Pin, C.: Estimating bacterial growth parameters by means of detection times. Appl. Enviro. Microbiol. 65(2), 732-736 (1999)

4. Baranyi, J., Roberts, T.A., McClure, P.: A nonautonomous differential-equation to model bacterial-growth. Food Microbio. 10(1), 43-59 (1993)

5. Baranyi, J., Roberts, T.A., McClure, P.: Some properties of a nonautonomous determistic growth-model describing the adjustment of the bacterial population to a new environment. IMA. J. Math. Appl. Med. Bio. 10(4), 293-299 (1993)

6. Edwards, M.P., Anderssen, R.S.: Symmetries and solutions of the non-autonomous von bertalanffy equation. Commun. Nonlinear Sci. Numer. Simulat. 22, 1062-1067 (2015)

7. Edwards, M.P., Schumann, U., Anderssen, R.S.: Modelling microbial growth in a closed environment. J. Math-for-Industry. 5, 33-40 (2013)

8. Peleg, M., Corradini, M.G.: Microbial growth curves: what the models tell us and what they cannot. Crit. Rev. Food Sci. Nutr. 51(10), 917-945 (2011)

9. Peleg, M., Corradini, M.G., Normand, M.D.: Isothermal and Non-isothermal Kinetic Models of Chemical Processes in Foods Governed by Competing Mechanisms. J. Agric. Food Chem. 57(16), 7377-7386 (2009)

10. Wardle, D.A.: A comparative-assessment of factors which influence microbial biomass carbon and nitrogen levels in soil. Biol. Rev. Camb. Philos. Soc. 67, 321-358 (1992)

\section{Submit your manuscript to a SpringerOpen ${ }^{\circ}$ journal and benefit from:}

- Convenient online submission

Rigorous peer review

- Immediate publication on acceptance

- Open access: articles freely available online

- High visibility within the field

- Retaining the copyright to your article

Submit your next manuscript at $>$ springeropen.com 